\title{
Melioidosis: A Fulminant Infection in a Patient with Uncontrolled Diabetes
}

\author{
Nandakishore Baikunje ${ }^{1}$ Suresh Gangaramajalu² Giridhar Belur Hosmane ${ }^{1}$ \\ ${ }^{1}$ Department of Pulmonary Medicine, K.S. Hegde Medical Academy, \\ NITTE (Deemed to be University), Mangaluru, Karnataka, India \\ ${ }^{2}$ Department of General Medicine, K.S. Hegde Medical Academy, \\ NITTE (Deemed to be University), Mangaluru, Karnataka, India

\begin{abstract}
Address for correspondence Nandakishore Baikunje, MBBS, MD, DM, Department of Pulmonary Medicine, K.S. Hegde Medical Academy, NITTE (Deemed to be University), Nityananda Nagara, Deralakatte, Mangaluru, Karnataka 575018, India (e-mail: nandanbaikunje@gmail.com).
\end{abstract}

J Health Allied Sci ${ }^{\mathrm{NU}}: 2021 ; 11: 50-53$

\begin{abstract}
Melioidosis is an endemic infection in Southeast Asia and Northern Australia commonly manifesting with pneumonia and localized skin infection. Though most exposures do not lead to severe illness, a fulminant infection can occur among patients with risk factors. A 59-year-old male presented with cough with expectoration and fever for 1 week. He had diabetes for 10 years with poorly controlled blood sugars. Contrast-enhanced computerized tomography (CECT) of thorax showed right upper lobe consolidation with diffuse ground-glass opacities in right upper lobe along with microabscesses in liver and spleen. Sputum culture and Xpert mycobacterium tuberculosis complex and resistance to rifampin (MTB/RIF) for tuberculosis were negative.

Keywords

- melioidosis

- Burkholderia

pseudomallei

- microabscess Bronchoalveolar lavage culture grew Burkholderia pseudomallei. He was treated with initial intensive therapy with injection amoxicillin-clavulanic acid for 2 weeks and subsequently started on eradication therapy with tablet trimethoprim-sulfamethoxazole. Diagnosis of melioidosis should be considered in a patient of pneumonia with multiorgan involvement in an endemic area, especially with underlying risk factors.
\end{abstract}

\section{Case History}

A 59-year-old male patient, an electrician by occupation, with exposure to wet season soil and water during commutation for work, presented with a history of intermittent fever for 1 week. It was associated with cough along with whitish-yellow sputum. There was no history of shortness of breath or chest pain. The patient was hailing from Western coastal Karnataka, an endemic region for melioidosis and had no travel history outside the area. He had diabetes for 10 years and was on oral metformin $500 \mathrm{mg}$ twice a day. His random blood sugar on admission was $323 \mathrm{mg} / \mathrm{dL}$, and HbA1c was $12.7 \%$. He had no other co-morbidities. He had a room air saturation of $97 \%$ on presentation. His chest X-ray showed illdefined nonhomogeneous consolidation in the right upper zone ( - Fig. 1). Sputum Gram stain showed numerous gram-positive cocci and gram-negative bacilli. Sputum culture yielded oropharyngeal flora. Sputum acid-fast bacillus (AFB) stain was negative. Blood culture did not show any organism. He was empirically started on intravenous amoxicillin-clavulanic acid $1.2 \mathrm{~g}$ thrice

published online

December 2, 2020
DOI https://doi.org/

10.1055/s-0040-1721232

ISSN 2582-4287. a day, and insulin injections were given for blood sugar control. A CECT thorax was done, which showed right upper lobe consolidation with diffuse ground-glass opacities of the right upper lobe ( - Fig. 2). Multiple enlarged mediastinal lymph nodes were seen ( $\boldsymbol{- \text { Fig. }} \mathbf{3}$ ) along with microabscesses in the liver and spleen. He underwent a bronchoscopy during which purulent secretions were seen coming from right upper lobe bronchus. Bronchoalveolar lavage was taken. AFB stain and Xpert MTB/RIF for tuberculosis were negative, and cytology showed numerous inflammatory cells. Culture of the bronchoalveolar lavage fluid in conventional blood culture medium grew Burkholderia pseudomallei. A sensitivity test was done using the Vitek-2 compact method, and the organism was found to be sensitive to amoxicillin/clavulanic acid, ceftazidime, imipenem, and trimethoprim/sulfamethoxazole. Since the patient was already receiving amoxicillin/clavulanic acid and was relieved of fever and cough by the time diagnosis was achieved, the same was continued for 2 weeks. He was started on eradication therapy with oral trimethoprim/

(C) 2020. Nitte (Deemed to be University).

This is an open access article published by Thieme under the terms of the Creative Commons Attribution-NonDerivative-NonCommercial-License, permitting copying and reproduction so long as the original work is given appropriate credit. Contents may not be used for commercial purposes, or adapted, remixed, transformed or built upon. (https://creativecommons.org/licenses/by-nc-nd/4.0/)

Thieme Medical and Scientific Publishers Pvt. Ltd., A-12, 2nd Floor, Sector 2, Noida-201301 UP, India 


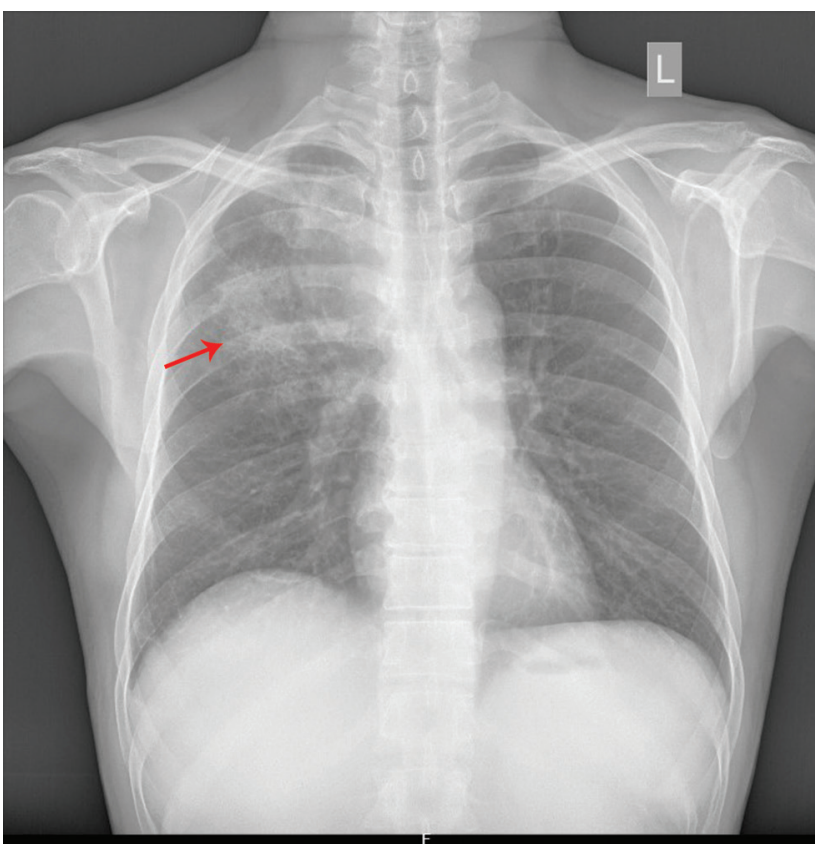

Fig. 1 Chest X-ray showing illdefined nonhomogenous consolidation in right upper zone.

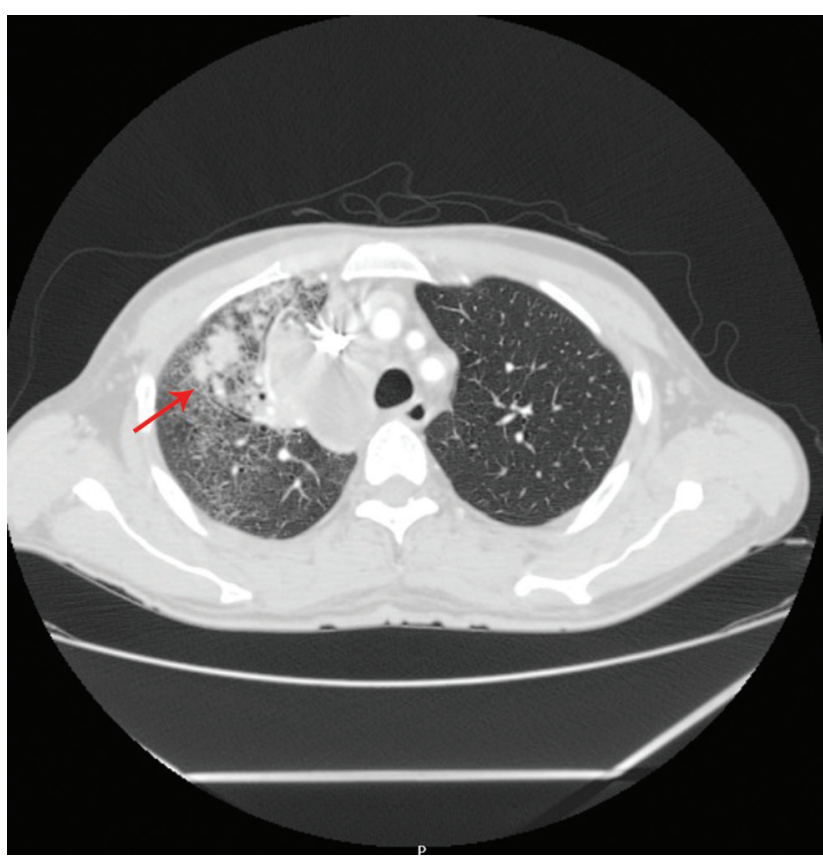

Fig. 2 CECT thorax (lung window) showing right upper lobe consolidation with diffuse ground glass opacities of the right upper lobe. CECT, contrast-enhanced computerized tomography.

sulfamethoxazole $(800 / 160 \mathrm{mg})$ two tablets twice a day with a plan to continue for 3 to 6 months. The patient was asymptomatic on follow-up at week 4 .

\section{Discussion}

Most studies indicate an increased risk of infection among people with diabetes compared with the general population. ${ }^{1,2}$ Risk of infection increases with poor

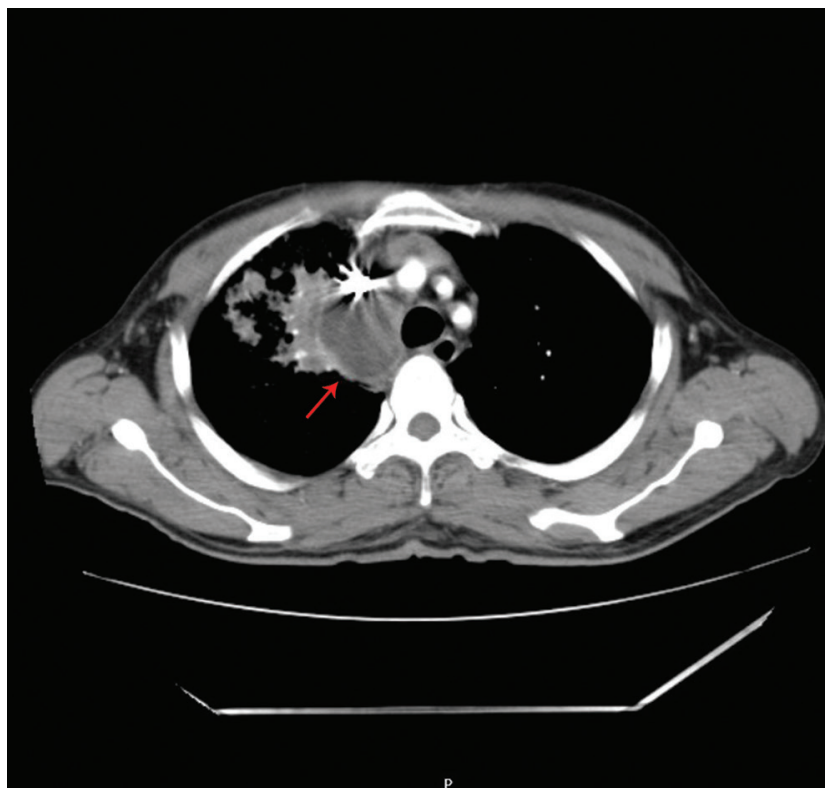

Fig. 3 CECT thorax (mediastinal window) showing multiple enlarged mediastinal lymph nodes. CECT, contrast-enhanced computerized tomography.

glycemic control. There are various host-related factors which predispose diabetic patients to infection, including hyperglycemia-related impairment of immune response, autonomic neuropathy, sensory neuropathy, vascular insufficiency, and skin and mucosal colonization with pathogens. Diabetics with hyperglycemia have depression of immune functions such as neutrophil chemotaxis, phagocytosis, intracellular bactericidal activity, opsonization, and cell-mediated immunity. ${ }^{3,4}$ Hence such patients are at risk for severe infections.

Awareness of the epidemiology of infection in the local community provides useful clues for diagnosis. Early diagnosis of the pathogen and appropriate treatment is the key to treatment success. Routine chest radiography and sputum sampling may fail to give an etiological diagnosis in many patients. It is necessary to pursue a microbiological diagnosis aggressively in diabetic patients with lung infiltrates. It helps in early directed therapy, simultaneously avoiding overly broad antimicrobial treatment.

Sputum samples should be sent for staining and culture whenever possible. Sometimes, induced sputum specimens may be required and most useful for diagnosing mycobacterial infections and pneumocystis pneumonia. ${ }^{5}$ In many cases, bronchoscopy and bronchoalveolar lavage are necessary to obtain adequate specimens. ${ }^{6}$ The sensitivity of bronchoalveolar lavage (BAL) is improved by early over late sampling and is reduced by ongoing or prior antimicrobial treatment. ${ }^{7,8} \mathrm{BAL}$ samples should be sent for microbiological studies (Gram stain/culture, AFB, Xpert MTB/RIF, KOH mount/fungal culture) to improve sensitivity.

B. pseudomallei is a facultative intracellular gram-negative bacterium responsible for causing melioidosis. ${ }^{9}$ It is a saprophyte distributed widely in soil and freshwater in endemic regions..$^{10}$ Infection is localized mainly to Southeast Asia and 
Northern Australia. ${ }^{11}$ Southern and Eastern states of India are considered endemic regions for melioidosis. ${ }^{12}$ The predominant mode of transmission is percutaneous inoculation from wet season soil or contaminated water. Other ways of transmission like inhalation, aspiration, and ingestion can also occur. ${ }^{13}$

Most exposures to B. pseudomallei do not result in illness in healthy individuals. Fulminant disease and fatalities can occur in those with defined risk factors. Most common risk factors for melioidosis are uncontrolled diabetes, alcoholism, chronic kidney disease, and chronic lung disease. ${ }^{14}$

Majority of endemic melioidosis occur during monsoon season. ${ }^{14}$ Index patient also presented in August during the peak of Southwest monsoon season in Western coastal Karnataka. This is attributed to increased exposure to soil and freshwater at work during the season.

Though most infections with B. pseudomallei result in nonsevere illness, immunocompromised hosts can have severe manifestations. ${ }^{15,16}$ Most common involvement are pneumonia and localized skin infections. Over half of patients of melioidosis can have bacteremia which can disseminate the organism to virtually any organ. ${ }^{15}$ Though it can affect people of any age; adults are commonly affected. Pneumonia is the most common manifestation in adults, whereas pneumonia and bacteremia are infrequently seen in children. ${ }^{17}$

An acute presentation with pneumonia may consist of high fever with chills and rigors, cough, sputum, and respiratory distress with or without shock. ${ }^{18}$ Chronic presentation may also be seen consisting of cough, purulent sputum production, hemoptysis, and night sweats closely resembling tuberculosis. Co-infection of melioidosis with tuberculosis has been reported previously. ${ }^{19}$ Presentation of melioidosis with mediastinal masses is described in literature. ${ }^{15}$ Our patient had necrotic mediastinal lymph nodes, raising further suspicion for tuberculosis. Investigations for tuberculosis were negative. Abscesses in internal organs like liver, spleen, kidney, and prostate are well recognized and should raise suspicion for melioidosis in endemic areas. ${ }^{20}$

Gram stain of sputum and abscess pus may reveal gram-negative bacilli of $B$. pseudomallei with characteristic bipolar staining with a "safety pin" appearance. However, culture is the mainstay of diagnosis. ${ }^{21}$ If initial sputum work-up is nondiagnostic, more invasive procedures like bronchoalveolar lavage may be required for the diagnosis.

Resistance to various antibiotics is well known, and treatment should be guided by culture sensitivity reports. ${ }^{22}$ Treatment should consist of an initial intensive phase with intravenous antibiotics for at least 2 weeks, followed by eradication therapy with oral antibiotics. Eradication therapy should be continued for at least 3 months as it helps in reducing the rate of relapse. ${ }^{23}$ Ceftazidime is the mainstay of intensive phase treatment, with carbapenems reserved for severe infections or treatment failures. Amoxicillin/clavulanic acid is used as second-line therapy. Trimethoprim/sulfamethoxazole (co-trimoxazole) is preferred for the eradication phase. ${ }^{24}$ However, treatment for individual patients should be adjusted according to clinical manifestations and response to treatment. Since index patient was already receiving amoxicillin/clavulanic acid when diagnosis was made and his symptoms had resolved and organism was found sensitive to amoxicillin/clavulanic acid, same was continued for intensive phase treatment. These patients require a close follow-up to observe for any relapse.

\section{Conclusion}

Melioidosis should be considered in the differentials when an immunocompromised patient presents with pneumonia and multisystem involvement. Microabscesses in internal organs are common. Infections are common among adults and in monsoon season in endemic areas. Culture is the mainstay of diagnosis. Appropriate samples should be sent for culture and may require invasive procedures like bronchoscopy for obtaining appropriate clinical samples if initial testing is nondiagnostic. Treatment consists of an initial intensive phase with intravenous antibiotics followed by prolonged eradication therapy with oral antibiotics as relapse is common.

\section{Conflict of Interest}

None declared.

\section{References}

1 Abu-Ashour W, Twells LK, Valcour JE, Gamble J-M. Diabetes and the occurrence of infection in primary care: a matched cohort study. BMC Infect Dis 2018;18(1):67

2 Carey IM, Critchley JA, DeWilde S, Harris T, Hosking FJ, Cook DG. Risk of infection in type 1 and type 2 diabetes compared with the general population: a matched cohort study. Diabetes Care 2018;41(3):513-521

3 Delamaire M, Maugendre D, Moreno M. Le Goff MC, Allannic H, Genetet B. Impaired leucocyte functions in diabetic patients. Diabet Med 1997;14(1):29-34

4 Hostetter MK. Handicaps to host defense. Effects of hyperglycemia on C3 and Candida albicans. Diabetes 1990;39(3):271-275

5 Fishman JA, Roth RS, Zanzot E, Enos EJ, Ferraro MJ. Use of induced sputum specimens for microbiologic diagnosis of infections due to organisms other than Pneumocystis carinii. J Clin Microbiol 1994;32(1):131-134

6 Brownback KR, Thomas LA, Simpson SQ. Role of bronchoalveolar lavage in the diagnosis of pulmonary infiltrates in immunocompromised patients. Curr Opin Infect Dis 2014; 27(4):322-328

7 Shannon VR, Andersson BS, Lei X, Champlin RE, Kontoyiannis DP. Utility of early versus late fiberoptic bronchoscopy in the evaluation of new pulmonary infiltrates following hematopoietic stem cell transplantation. Bone Marrow Transplant 2010;45(4): 647-655

8 Marr KA, Laverdiere M, Gugel A, Leisenring W. Antifungal therapy decreases sensitivity of the Aspergillus galactomannan enzyme immunoassay. Clin Infect Dis 2005;40(12):1762-1769

9 White NJ. Melioidosis. Lancet 2003;361(9370):1715-1722

10 Dance DA. Ecology of Burkholderia pseudomallei and the interactions between environmental Burkholderia spp. and human-animal hosts. Acta Trop 2000;74(2-3):159-168

11 Cheng AC, Currie BJ. Melioidosis: epidemiology, pathophysiology, and management. Clin Microbiol Rev 2005;18(2):383-416

12 Tipre M, Kingsley P, Smith T, Leader M, Sathiakumar N. Melioidosis in India and Bangladesh: a review of case reports. Asian Pac J Trop Med 2018;11(5):320 
13 Currie BJ, Fisher DA, Howard DM, et al. The epidemiology of melioidosis in Australia and Papua New Guinea. Acta Trop 2000;74(2-3):121-127

14 Suputtamongkol Y, Chaowagul W, Chetchotisakd P, et al. Risk factors for melioidosis and bacteremic melioidosis. Clin Infect Dis 1999;29(2):408-413

15 Currie BJ, Ward L, Cheng AC. The epidemiology and clinical spectrum of melioidosis: 540 cases from the 20 year Darwin prospective study. PLoS Negl Trop Dis 2010;4(11):e900

16 McLeod C, Morris PS, Bauert PA, et al. Clinical presentation and medical management of melioidosis in children: a 24-year prospective study in the Northern Territory of Australia and review of the literature. Clin Infect Dis 2015;60(1):21-26

17 Birnie E, Virk HS, Savelkoel J, et al. Global burden of melioidosis in 2015: a systematic review and data synthesis. Lancet Infect Dis 2019;19(8):892-902

18 Meumann EM, Cheng AC, Ward L, Currie BJ. Clinical features and epidemiology of melioidosis pneumonia: results from a 21-year study and review of the literature. Clin Infect Dis 2012; 54(3):362-369

19 Garg R, Shaw T, Vandana KE, Magazine R, Mukhopadhyay C. Melioidosis in suspected recurrent tuberculosis: a disease in disguise. J Infect Dev Ctries 2020;14(3):312-316

20 Currie BJ, Fisher DA, Howard DM, et al. Endemic melioidosis in tropical northern Australia: a 10-year prospective study and review of the literature. Clin Infect Dis 2000;31(4):981-986

21 Hoffmaster AR, AuCoin D, Baccam P, et al. Melioidosis diagnostic workshop, 2013. Emerg Infect Dis 2015;21(2):e141045

22 Eickhoff TC, Bennett JV, Hayes PS, Feeley J. Pseudomonas pseudomallei: susceptibility to chemotherapeutic agents. J Infect Dis 1970;121(2):95-102

23 Chaowagul W, Suputtamongkol Y, Dance DA, Rajchanuvong A Pattara-arechachai J, White NJ. Relapse in melioidosis: incidence and risk factors. J Infect Dis 1993;168(5):1181-1185

24 Dance D. Treatment and prophylaxis of melioidosis. Int J Antimicrob Agents 2014;43(4):310-318 